\title{
A Mechanism-Based Approach to P2X7 Receptor Action
}

\author{
Mehmet Ugur and Özlem Ugur \\ Department of Biophysics (M.U.) and Department of Pharmacology (O.U.), Faculty of Medicine, Ankara University, Ankara, \\ Turkey
}

Received October 25, 2018; accepted January 30, 2019

\begin{abstract}
The ligand-gated ion channel P2X7 receptor attracts special attention due to its widespread presence as well as its unusual responses. Besides relatively well-understood mechanisms such as intracellular $\mathrm{Ca}^{2+}$ increase and $\mathrm{K}^{+}$depletion, the P2X7 receptor activates other peculiar responses whose mechanisms are not fully understood. The best known among these is the permeabilization of the cell membrane to large molecules. This permeabilization has been explained by the activation of a nonselective permeation pathway by the P2X7 receptor, a phenomenon called "pore formation." However, with the emergence of new data, it became apparent that large molecules enter the cell directly through the pore of the ion channel, similar to the smaller ions. This explanation seems to be true for cationic large molecules. On the other hand, there is still convincing evidence indicating that
\end{abstract}

the $\mathrm{P} 2 \mathrm{X} 7$ receptor activates a separate pathway that permeates anionic large molecules in some cell types. Furthermore, there exist functional data suggesting that the P2X7 receptor may also activate other intracellular signaling molecules or other ion channels. Interestingly and contrary to what is expected from a ligand-gated channel, these activations occur in a seemingly direct manner. Somewhat overshadowed by the pore formation hypothesis, these action mechanisms may lead to a better understanding of not only the P2X7 receptor itself but also some important physiologic functions such as the release of anionic autocoids/neurotransmitters in the central nervous system. This review discusses, assesses, and draws attention to the data concerning these neglected but potentially important points in the $\mathrm{P} 2 \mathrm{X} 7$ receptor field.

\section{Introduction}

The $\mathrm{P} 2 \mathrm{X} 7$ receptor (P2X7R) belongs to the $\mathrm{P} 2 \mathrm{X}$ receptor family, all seven members (P2X1-7) of which are widely accepted as ligand-gated ion channels that can be activated by extracellular ATP. However, this receptor is known to have other peculiar actions that distinguish it from other ligand-gated channels. Along with molecular interventions including knockout of the receptor, vast amount of experimental evidence based on pharmacologic experiments with specific agonists and antagonists show that P2X7R is involved in a diverse set of cellular responses.

Recent interest in P2X7R field has concentrated mostly around areas of cancer and inflammation. ATP has been seen as an important mediator in inflammation and tumor biology as its concentration can reach high levels around the cells in an inflamed area or inside a tumor, thus potentially providing

This work is sponsored by the Scientific and Technological Council of Turkey (Grant number 113S376).

https://doi.org/10.1124/mol.118.115022. a sustained background stimulus regulating and affecting the function of immune and neoplastic cells. With its relatively low sensitivity to ATP, P2X7R is a good candidate for a receptor that will function in such a high-ATP environment. Concordantly, several studies have suggested that P2X7R is an important, even a central player in key processes related to inflammation and cancer such as the activation of interleukin $1 \beta$ (IL-1 $\beta$ ) processing most probably via NLRP3 (nucleotidebinding oligomerization domain, leucine rich repeat, and pyrin domain containing 3) inflammasome complex in macrophagelike immune cells (Ferrari et al., 1997a; Solle et al., 2001; for details, see Adinolfi et al., 2018), in vivo growth (Adinolfi et al., 2012; Amoroso et al., 2015), and invasion of cancer cells (Ferrari et al., 2006; Xia et al., 2015; Di Virgilio and Adinolfi, 2017; Di Virgilio et al., 2017).

As we shall see throughout this review, involvement of P2X7R in physiology and pathophysiology is actually much wider than these examples suggest. On the other hand, despite the vast amount of effort put in the field, the mechanisms of action for P2X7R are still not well understood. Therefore, this

ABBREVIATIONS: AKT, protein kinase B; HEK293, human embryonic kidney cells; IL-1 $\beta$, interleukin $1 \beta$; LDH, lactate dehydrogenase enzyme; $\mathrm{Ni}$-AchR, nicotinic acetylcholine receptor; NLRP3, nucleotide-binding oligomerization domain, leucine rich repeat, and pyrin domain containing 3; NMDG, N-methyl-D-glucamine; PLA2, phospholipase A2 enzyme; PLD, phospholipase D enzyme; P2X2(4,7)R, P2X purinergic receptor 2(4,7); ToTo-1, 1,1'-(4,4,7,7-tetramethyl-4,7-diazaundecamethylene)-bis-4-(3-methyl-2,3-dihydro-(benzo-1,3-thiazole)-2-methylidene)-quinolinium tetraiodide; TRPV, transient receptor potential cation channel V; YoPro-1, 4-((3-methyl-2(3H)-benzoxazolylidene)methyl)-1-(3-(trimethylammonio)propyl) quinolinium diiodide. 
review's main focus will be on the mechanisms of action that P2X7R uses to activate cellular responses rather than on what those responses are or what pathologic processes this receptor is involved in. We will use examples of physiologic/pathologic phenomena where P2X7R is thought to play important roles only with the intention of illustrating our points and giving an idea of the implications of the mechanisms that we discuss in the review. For a general view of the structure, pharmacology, or the wide range of physiologic and pathologic actions of P2X7R, the following comprehensive reviews are recommended: Di Virgilio and Adinolfi (2017); Di Virgilio et al. (2017, 2018); Adinolfi et al. (2018); Peverini et al. (2018); and Young and Górecki (2018).

Since the initial observations made in mast and transformed 3T3 cells (Rozengurt et al., 1977; Cockcroft and Gomperts, 1980) and later in macrophages (Steinberg et al., 1987), ATP has been known to induce uptake of both the cationic and the anionic large organic molecules such as fluorescent dyes into the cells as well as to release of some metabolites (mostly anionic) from the cell. The hypothetical ATP receptor that mediates this permeabilization response was initially called P2Z. Later, the cloned ligand-gated channel protein P2X7R was identified to be this ATP receptor (Surprenant et al., 1996).

In due course, it was postulated that P2X7R permeabilized cells to large molecules either by dilation of its own pore (Surprenant et al., 1996) or activation of another nonselective large-pore-forming protein (Pelegrin and Surprenant, 2006). This mechanism was named "large pore formation" (or simply "pore formation"), and P2X7R was thought to be unique in this regard. For a long time, "large pore formation" had been the central mechanism to explain P2X7R actions. However, P2X7R does not appear to be unique in increasing membrane permeability to large molecules nor does it seem to activate large pores, as will be discussed later in detail. In light of recent experimental findings, the P2X7Rinduced permeability to large molecules is now thought to be simply a direct result of the opening of the P2X7R cation channel.

It is our belief that neither the debate on permeation to large molecules nor the issues regarding other unique interactions of P2X7R have reached a satisfactory conclusion. Moreover, the overemphasis on the pore formation mechanism obscured the data that could hold important clues for the probable mechanisms of P2X7R action, which may be far more diverse than what was thought before. This review presents and discusses these mechanisms in a clear way. We will form testable working hypotheses based on a comprehensive review and a detailed discussion of the available data. Although we will discuss all the aspects of P2X7R action mechanisms, we believe that two mechanisms not prominently covered in the literature need special attention. The first is the less-appreciated mechanism for large molecule permeation. The second is the possible direct interaction of the P2X7R with intracellular signaling pathways.

In general, three not mutually exclusive mechanisms of action are proposed for P2X7R:

1. As a regular ligand-gated ion channel, the receptor, upon stimulation, may increase the cell membrane permeability to small cations, leading to depolarization of the cell membrane, $\mathrm{Ca}^{2+}$ uptake into the cell, or $\mathrm{K}^{+}$ efflux from the cell.
2. It may increase the permeability of the cell membrane to large molecules that are too large to go through regular ion channels, leading to either uptake or loss of big molecules such as nucleotides. As mentioned before, the so-called pore formation or cytolytic action has been the most popular mechanism to explain this permeability. It was proposed to happen either through a gradual dilation of the ion channel pore or activation of an accessory protein that can conduct large molecules.

3. Through direct interaction, it may activate some intracellular signal transduction proteins and cellular processes.

Mechanism 1: Is P2X7R a Ligand-Gated Cation Channel? There is little doubt that P2X7R is a ligand-gated cation-channel. However, whether it is a regular cation-channel similar to the nicotinic acetylcholine receptor (Ni-AchR) is quite controversial. Because, in the course of stimulation, it seems to permeate large cationic and also anionic molecules, which normally should not go through ion channels. The question as to whether P2X7R ion channel goes through a "pore dilation" process for this permeation to occur will be discussed later. Nevertheless, as P2X7R activation is known to cause a permeability increase to anionic molecules as well, the first question to ask is whether P2X7R really forms a cation-selective conduit or is a nonselective channel (i.e., permeates both cations and anions).

Interestingly, the results of the electrophysiological experiments concerning this question fall into two categories according to the experimental system used. The first category contains the results obtained in heterologous expression systems. In electrophysiological experiments conducted in expression systems that do not express the receptor endogenously, P2X7R activation mostly leads to a cation-selective whole-cell current (Virginio et al., 1999a) and a cation-selective single channel activity, with no significant selectivity between small cations such as $\mathrm{Na}^{+}, \mathrm{K}^{+}$, or $\mathrm{Ca}^{2+}$ (Riedel et al., 2007a,b). The P2X7R permeation ratio for $\mathrm{Cl}^{-}: \mathrm{Na}^{+}$was reported to be about 1:100 or less. Thus, compared with cations this ionic current/channel is almost impermeable to anions, including $\mathrm{Cl}^{-}$(Virginio et al., 1999a; Kubick et al., 2011; Browne et al., 2013). P2X7R current has been shown to be carried not only by $\mathrm{Na}^{+}, \mathrm{K}^{+}$, and $\mathrm{Ca}^{2+}$ but also by large organic cations such as $\mathrm{NMDG}^{+}$ (N-methyl-D-glucamine) or Tris ${ }^{+}$, the largest of those being tetraethylammonium $^{+}$(130 Da) (Riedel et al., 2007a,b).

However, as expected, the large cations have smaller permeabilities. The second category comprises the results obtained in cells that naturally express the P2X7R such as mast cells and astrocytes. The examples in this category are intriguing but unfortunately few. In these cells, ATP stimulation seems to activate a nonselective current, conducting both anions (including $\mathrm{Cl}^{-}$, glutamate ${ }^{-}$, and aspartate ${ }^{-}$) and cations in the wholecell mode (Tatham and Lindau, 1990; Duan et al., 2003).

As mentioned previously, the other important question concerning the P2X7R is whether its activation gradually increases the permeability of the membrane through either enlargement of receptor's own ionic pore or the activation of another "pore" protein to allow the passage of molecules that are usually too large to pass through the ion channels. In other words, does pore formation occur?

Pore formation theory was based on the initial electrophysiologic observation that prolonged activation of P2X7R led to a time-dependent change in reversal potential of the whole-cell 
currents; a gradual shift from more negative to significantly less negative values (Surprenant et al., 1996). Assessment of reversal potentials of ionic currents is the usual method to determine the relative permeability of ion channels to different ions. These measurements are conducted under conditions where the ions such as $\mathrm{Na}^{+}$or $\mathrm{K}^{+}$are replaced with large organic molecules with a similar charge $\left(\mathrm{NMDG}^{+}\right.$or Tris $\left.{ }^{+}\right)$. This so-called ion replacement is done asymmetrically-that is, usually only in the bathing solution.

According to the Goldman-Hodgkin-Katz equation, two factors act together to determine the reversal potential of the membrane current; the relative permeabilities of the ions and the ratio of their extracellular to intracellular concentrations (i.e., Nerst potential of the ion). For the regular ion channels, the reversal potential of the current is expected to be a negative value under the asymmetric ionic replacement conditions described previously because the large ions such as $\mathrm{NMDG}^{+}$have no significant permeation; thus, the current is mostly determined by the small ions $\left(\mathrm{Na}^{+}\right.$or $\left.\mathrm{K}^{+}\right)$, which have negative Nernst potentials.

As expected, a negative reversal potential was observed for the whole-cell current at the initial phase of the P2X7R activation. Unexpectedly, however, the reversal potential gradually changed from negative values toward zero. This wellrepeated observation was interpreted as an indication of P2X7R channel becoming more permeable to large organic cations (i.e., opening of a large nonselective pore). Let us reiterate that this proposed large nonselective pore opening does not mean that the P2X7R current becomes nonselective between cations and anions; the current seems to stay cation-selective and does not permeate anions. It is thought to lose its selectivity between large and small cations and gradually become more permeable to large cations (Virginio et al., 1999a). Let us also state that this gradual shift in the reversal potential was later found not to be unique to P2X7R and has been described for other ligand-gated ion channels such as P2X2R, P2X4R (Khakh et al., 1999; Virginio et al., 1999b), and transient receptor potential cation channel V1 (TRPV1) (Chung et al., 2008).

In the case of P2X7R, enlargement of the receptor's pore itself was proposed to explain this permeability increase to large cations. However, more recent single-channel experiments have given results that are quite inconsistent with this ion channel-to-pore enlargement idea. Single-channel data suggest that, in contrast to the whole-cell currents, P2X7R single-channel currents have a very stable reversal potential, as expected from a regular ion channel. Nevertheless, the P2X7R channel is somewhat more permeable to large cations than many other ion channels are. This is the case from the beginning of its activation; its permeability characteristics do not change over time (Riedel et al., 2007a,b, see also Harkat et al. (2017) for a similar finding in P2X2R). Furthermore, data obtained by cysteine accessibility mutations suggest that P2X7R conduction pore does not show any sign of dilation during receptor activation (Pippel et al., 2017).

Even if the single-channel data indicate that P2X7R pore itself does not enlarge, it is still possible that an additional conduction pathway that is gradually activated by the receptor in the whole-cell settings may increase the permeability to large cations. However, a recent study elegantly and convincingly demonstrated that this is also not the case, and the reversal shift observed in the whole-cell setting is likely to be an artifact ( $\mathrm{Li}$ et al., 2015). In the ion replacement experiments where the reversal potential shift was observed, the ionic concentrations of the intracellular and extracellular solutions were assumed to be stable throughout the experiment. Hence, the observed reversal potential change was attributed to an increase in the permeability to large ions.

However, the study by $\mathrm{Li}$ et al. (2015) shows that this assumption is not justified in the case of P2X2R. The intracellular concentration of the large cations used in the extracellular solution for the ion replacement is gradually increased due to the large and persistent P2X2R-activated membrane conductance. This increase is the result of the small but measurable permeation of these large cations through the P2X2R channel. Additionally, intracellular small ion concentration decreases during P2X2R activation. Thus, after the findings of Li et al. (2015), the reversal potential shift can very well be explained by the change in the composition of the intra-cellular solution rather than an increased permeability to large cations.

Even though the observation was made for P2X2R, the main argument is relevant for all the receptors assumed to exhibit pore dilation, including P2X7R. Hence, for P2X7R there seems to be no pore dilation or pore activation but simply the opening of a cation-channel that is somewhat permeable to large cations. A more detailed discussion of the subject can be found in Peverini et al. (2018). Additionally, the idea that proteins such as pannexins activated by P2X7R form a pathway that permeates large cationic molecules (Pelegrin and Surprenant, 2006) was not confirmed by later studies (e.g., Qu et al., 2011; Hanley et al., 2012; Alberto et al., 2013).

Thus, the experimental results discussed in this section lead to three general conclusions: 1) When heterologously expressed, P2X7R forms a ligand-gated ion channel that is strictly cationselective and, besides small cations, permeates larger organic cations such as tetramethylammonium. 2) The selectivity of the P2X7R-activated current does not change with time and terms like "pore dilation," "channel to pore transition," or "pore activation" are not correct descriptions of what actually happens. 3) Despite the facts in 1) and 2), P2X7R is able to induce a nonselective whole-cell current that permeates both cations and anions in certain cell types that naturally express the receptor.

Ligand-gated ion channel activity is the best established mechanism for P2X7R. Being a cation-channel, P2X7R can cause $\mathrm{Ca}^{+2}$ influx and increase intracellular $\mathrm{Ca}^{+2}$ concentrations, as shown by numerous studies. Intracellular $\mathrm{Ca}^{+2}$ increase was clearly shown to be the mechanism responsible for a number of P2X7R-induced responses, as in the case of protein kinase B (AKT) activation in astrocytes (Jacques-Silva et al., 2004). In other cases, however, involvement of the P2X7R's ion channel activity is not so clear-cut. For example, as mentioned previously, NLRP3 complex-related IL-1 $\beta$ processing, an apparently crucial step in inflammation, is triggered by P2X7R. $\mathrm{K}^{+}$efflux (more precisely, the decrease of intracellular $\mathrm{K}^{+}$concentration due to this efflux) was proposed to be the trigger for this response (Walev et al., 1995; Ferrari et al., 1997a). Because it readily permeates $\mathrm{K}^{+}$ions and does not inactivate easily, P2X7R is a fairly suitable conduit for this role, and the $\mathrm{K}^{+}$depletion was initially thought to occur through the P2X7R channel (for details, see Di Virgilio et al. (2017), Adinolfi et al. (2018)).

However, a recent study suggests that besides P2X7R another ion channel, namely the TWIK2 $\mathrm{K}^{+}$channel, is also essential for NLRP3 complex-related IL-1 $\beta$ processing (Di et al., 2018). 
This study showed that, in macrophages, the TWIK2 channel could clearly add a substantial $\mathrm{K}^{+}$conductivity to what could be provided by P2X7R alone. TWIK2-provided $\mathrm{K}^{+}$current seems to be essential for P2X7R-induced IL-1 $\beta$ processing because this response is absent in TWIK2 knockout Kcnk6 ${ }^{-/-}$ macrophages or in macrophages where TWIK2 is knocked down by using small-interfering RNA. Interestingly, however, this channel appears to be activated not by P2X7R but by another, as-yet-unidentified ATP receptor because TWIK2 currents can readily be activated by ATP in P2X7R $\mathrm{R}^{-1-}$ macrophages (Di et al., 2018). If $\mathrm{K}^{+}$efflux (intracellular $\mathrm{K}^{+}$depletion) and P2X7R activation are both essential for ATP-induced IL-1 $\beta$ processing, as suggested by various studies, then according to the results of Di et al. (2018) it seems that the role of P2X7R in IL- $1 \beta$ processing is not increasing the $\mathrm{K}^{+}$conductance.

P2X7R must then serve some other essential, yet unknown function. As Di et al. (2018) pointed out, this function can be to provide a $\mathrm{Na}^{+}$conductance to counter the hyperpolarizing effect of the increased $\mathrm{K}^{+}$conductance, because hyperpolarization paradoxically diminishes the electrochemical potential driving the $\mathrm{K}^{+}$efflux. As can be seen from this discussion, the action mechanism of P2X7R in IL- $1 \beta$ processing is still not fully understood, and further investigation is needed.

Mechanism 2. Does P2X7R Permeabilize the Cells to Large Molecules? As discussed earlier, the answer to the question of whether P2X7R permeabilizes the cells to large molecules is yes. Uptake of fluorescent dyes into cells is the most preferred tool to assess the permeability of cells to large molecules, and P2X7R stimulation is well known to cause cell membranes to become permeable to fluorescent dyes. These dyes are considerably larger than the cationic molecules such as $\mathrm{NMDG}^{+}$that are used in electrophysiologic studies.

Before proceeding further, one point must be clarified here. With long P2X7R stimulations lasting for a few hours, cell death and other drastic and complex processes may permeabilize the cells to molecules as large as lactate dehydrogenase enzyme (LDH) (e.g., Ferrari et al., 1997b; Le Stunff and Raymond, 2007). The P2X7R-induced permeabilization process that we are referring to should be distinguished from this type of permeabilization. What we are interested in here is a permeabilization that starts almost immediately after the P2X7R stimulation. We will be discussing observations lasting in the range of 10-20 minutes rather than hours and regarding the phenomenon that renders the plasma membrane permeable to molecules not larger than $1000 \mathrm{Da}$.

Thus, the "large molecules" in question here are considerably smaller compared with LDH (molecular mass 140,000 Da). That said, we can now focus on an interesting property of the P2X7R-induced cell permeabilization. Similar to the ionic currents, the selectivity of the dye-uptake phenomenon also seems to be dependent on the experimental system used. P2X7Rstimulated uptake seems to be selective for cationic-dyes in heterologous expression systems but tends to be nonselective when P2X7R is endogenously expressed in the cell.

Numerous observations clearly have shown that when a functioning P2X7R is externally expressed, a cationic dye (such as ethidium bromide or YoPro-1 [4-((3-methyl-2(3H)benzoxazolylidene)methyl)-1-(3-(trimethylammonio)propyl)quinolinium diiodide]) uptake occurs (Surprenant et al., 1996; Virginio et al., 1999a and many others). By contrast, the number of the studies that systematically have investigated the permeation of anionic dyes by the activation of heterologously expressed P2X7R is limited. Nevertheless, two studies conducted in human embryonic kidney 293 (HEK293) cells expressing a transfected P2X7R, despite using very different experimental desgns, showed clearly that P2X7R activation does not cause any anionic dye uptake. In both studies, while cationic dyes as large as ToTo-1 (894 Da, 1,1'-(4,4,7,7-tetramethyl-4,7diazaundecamethylene)-bis-4-(3-methyl-2,3-dihydro-(benzo-1,3thiazole)-2-methylidene)-quinolinium tetraiodide) could easily pass through, anionic ones such as lucifer yellow (433 Da), fluorescein (332 Da), or calcein (623 Da) were clearly excluded from the P2X7R-activated permeation pathway (Schachter et al., 2008; Cankurtaran-Sayar et al., 2009). Although a $\mathrm{Ca}^{2+}$-activated lucifer yellow uptake was also observable, this was not a P2X7R-dependent uptake as it could also be activated by a calcium ionophore in parent HEK293 cells, and it was possibly due to a $\mathrm{Ca}^{2+}$-activated anion channel (Cankurtaran-Sayar et al., 2009).

This brings us to the question of whether these large cationic dyes go through the P2X7R channel itself, as the small cations do, or through another permeation pathway. Cationic dye uptake and cation channel activity have been claimed to be dissociated by performing mutations or deletions (especially at the carboxy terminal) on the P2X7R receptor or by expressing it in oocytes. Thus, at first sight, separate permeation pathways seem to exist for small cations and large cationic dyes. However, there are problems associated with this body of data, which can be summarized as follows. Deletion of a cysteine-rich region in the carboxyl terminal of the protein, which was shown to reduce the ionic currents and the YoPro-1 uptake induced by P2X7R (Surprenant et al., 1996), was later claimed to abolish the reversal potential shift but not the YoPro-1 uptake (Jiang et al., 2005).

Contrary to this finding, Yan et al. (2008) found that the same deletion did not affect the reversal potential shift observed with P2X7R currents. A similar but naturally occurring truncation was also reported to abolish only the YoPro-1 uptake but not the ionic currents (Adinolfi et al., 2010). Yet, on a closer look, what this mutation seems to do in the study by Adinolfi et al. (2010) is simply to reduce the small $\left(\mathrm{Ca}^{2+}\right)$ and large (YoPro-1) cation uptake in a comparable way; in conjunction with the results of a previous study performed with another carboxyl terminal truncated P2X7R (Adinolfi et al., 2005), it looks as though this truncation reduces the overall activity of the receptor. Such a loss in P2X7R activity with carboxyl tail deletion is also suggested by the findings of Karasawa et al. (2017).

In another study, carboxyl terminal truncation of P2X7R was claimed not to affect the ionic currents but abolish the dyeuptake (Smart et al., 2003). However, the use of different cell types to measure ionic currents (oocytes) and the dye uptake (HEK-293) seems to be a major drawback of this study, as the magnitude of the functional loss caused by the truncation was later shown to change in a complicated way, depending on the membrane lipid composition or the cell type (Karasawa et al., 2017).

A similar argument can also be made for the effect of E496A mutation on P2X7R: This mutation was claimed to affect the dye uptake and ionic currents differentially as it abolished the cationic dye uptake in one study (Gu et al., 2001) but did not affect the ionic currents in another (Boldt et al., 2003). However, only a single type of response was measured in each study, and in neither of them was the state of the other response checked. 
A loss of function due to carboxyl terminal truncation (or other alterations), affecting the large and the small cation permeation in a similar way is expected to reduce the dye uptake and, according to $\mathrm{Li}$ et al. (2015), also diminish the reversal potential shift. It appears that such a loss of function may explain the discrepant results given for the truncated P2X7R, especially if this function loss is cell type-dependent, as suggested by Karasawa et al. (2017). Still, why the reversal potential shift and dye uptake were affected in such a different way in Jiang et al. (2005) cannot be explained easily by this argument.

The lack of reversal potential shift with P2X7R-activated ionic currents when the protein is expressed in oocytes was also interpreted to indicate that P2X7R induced large and small ion permeability through separate pathways (Petrou et al., 1997). However, when the unusually short duration of the inactivating P2X7R current that they presented and the large volume of the oocyte are taken into consideration, the model proposed by Li et al. (2015) exactly predicts the lack of the reversal potential shift in the study of Petrou et al. (1997).

On the other hand, there exists an alternative group of data suggesting that large and small cations go through the same pore. When purified and reconstituted into lipid vesicles, carboxyl terminal truncated P2X7R, by itself, was sufficient for the uptake of the cationic dye YoPro-1 as well as $\mathrm{Ca}^{2+}$, and changing the lipid composition of the reconstitution vesicles, especially the cholesterol content, affected YoPro- 1 and $\mathrm{Ca}^{2+}$ permeations in a similar way (Karasawa et al., 2017). This direct finding strongly supports the hypothesis that at least the cationic-dyes can permeate through the P2X7R channel.

In another interesting study, where the dye uptake and the ionic currents were measured simultaneously in voltageclamped single cells, it was shown that the cationic dye uptake was in lockstep with the P2X7R-activated ionic current in terms of both the time course and the current (flux)/voltage characteristics. Additionally, when the cation selectivity of the channel was altered by mutations on the P2X7R protein pore region, the permeability to both $\mathrm{Cl}^{-}$and negative dyes increased together, suggesting that small and large ions (dyes) go through the same pore (Browne et al., 2013). In conclusion, the experimental evidence supporting the hypothesis that the cationic dye uptake is through the P2X7R channel itself, compared with those in favor of separate permeation pathways, seems to be more direct and internally coherent, which biases us toward the idea that a single permeation pathway exists for all cations (see also Peverini et al. (2018)). Here, it should be mentioned again that P2X7R is not unique in being permeable to large cationic dyes; other ligand-gated cationic channels such as P2X2, P2X4 (Khakh et al., 1999; Virginio et al., 1999b), TRPV1 (Chung et al., 2008), and TRPA1 (Banke et al., 2010) can induce the uptake of large cationic dyes into cells.

Compared with the expression systems, the cells that naturally express P2X7R exhibit a very different picture in terms of dye permeability. It is well known that ATP/BzATP application to macrophages, astrocytes, or macrophage-derived cell lines such as J744 or RAW264 causes permeability not only to cationic dyes like YoPro-1 (Pelegrin and Surprenant, 2006; Le Stunff and Raymond, 2007; Schachter et al., 2008; Cankurtaran-Sayar et al., 2009) but also to anionic dyes such as lucifer yellow (433 Da), fluorescein (332 Da), calcein (623 Da) (Steinberg et al., 1987; Duan et al., 2003; Fellin et al., 2006; Schachter et al., 2008; Cankurtaran-Sayar et al., 2009), indo-1 (650 Da) (Greenberg et al., 1988), and fura-2 (831 Da) (Steinberg et al., 1987).This anionic dye permeability is reversible because it ceases when extracellular ATP is washed away (Steinberg et al., 1987; Schachter et al., 2008; Cankurtaran-Sayar et al., 2009). It is definitely not through pinocytosis (Steinberg et al., 1987) or not due to intracellular $\mathrm{Ca}^{2+}$ increase or $\mathrm{K}^{+}$loss from the cells (Cankurtaran-Sayar et al., 2009). Hence, if the ATP receptor in these cells is indeed P2X7R, as the agonist and antagonist specificity data suggest, then the anionic dye uptake by P2X7R stimulation seems to be cell-type dependent (occurs in macrophages but not in HEK293 cells, for example).

There is one other study with anecdotal evidence that should be mentioned here. In neuron-derived GT1 cells, stimulation of externally expressed P2X7R seems to cause a clear leakage of the preloaded anionic dyes fura-2 and fura-TT out of the cell, as well as a cationic dye uptake (Yan et al., 2008). This finding further suggests the cell-type dependence of the anionic dye permeation.

Thus, together with the electrophysiologic results presented in the previous section we may conclude that:

1. Externally transfected P2X7R activates a cation-selective ionic current and also a cation-selective dye uptake.

2. The P2X7R ion channel seems to permeate large cations like tetraethylammonium ${ }^{+}$as well as larger cationic dyes like YoPro-1.

Working hypotheses about action mechanisms of P2X7R :

1) $P 2 X 7 R$ is a ligand-gated cation-channel that permeates large cationic molecules as big as the dye Yo-Pro but not anions such as lucifer yellow or even $\mathrm{Cl}^{-}$. In this sense, it is similar to other ligand-gated channels such as P2X2R and TRPs.

2) $P 2 X 7 R$ activates an accessory pathway which permeates anionic large molecules. This unidentified permeation pathway exists only in some cell types and can be an anion transporter or an ion channel (Figure 2, first model). Its activation does not involve mechanisms related to the cation-channel activity of the receptor such as $\mathrm{Ca}^{2+}$ influx, $\mathrm{K}^{+}$depletion or depolarization.

3) P2X7R, in addition to its ligand-gated ion channel activity, can also interact directly with intracellular signaling pathways and activate other ion channels, enzymes and the anionic permeation pathway mentioned in the second hypothesis.

Fig. 1. Working hypotheses about the action mechanisms of P2X7R. 
3. In cells that naturally express P2X7R, the receptor activates a nonselective current and a nonselective dye uptake, through which both anionic and cationic molecules can pass.

4. Additionally, as discussed in the previous section, the evidence for P2X7R-induced nonselective large molecule uptake mechanisms such as the pore dilation or the involvement of accessory pore proteins is not very strong.

The simplest model to encompass all these facts would be to assume that the cation-specific P2X7R ion channel simply permeates small cations and also, to some extent, large cationic molecules such as cationic dyes. (First working hypothesis in Fig. 1) However, in this case, to explain the permeability for large anionic molecules that is observed in certain cell types, we must admit a cell type-dependent presence of a separate P2X7R-activated permeation pathway that is anion specific. (Second working hypothesis in Fig. 1, model 1 in Fig. 2, as opposed to the alternative model 2 in Fig. 2.) This hypothesis also explains why the $\mathrm{Cl}^{-}$currents are observed exclusively in cells that naturally express P2X7R (Tatham and Lindau, 1990; Duan et al., 2003).

The hypothesis about a separate and cell-dependent pathway for anionic dyes is further supported by the observation that although all of the RAW264 cells in a population uptake the cationic dye ethidium bromide, only some of them can uptake the anionic dye lucifer yellow (Cankurtaran-Sayar et al., 2009). This observation, which was confirmed by Young and Górecki (2018) in dystrophic myoblasts, indicates a cell-to-cell variability across a cell population in terms of the anionic uptake phenomenon.

Setting the mechanism(s) aside for now, it is an observable fact that P2X7R induces uptake of anionic large molecules as well as cationic ones. What function, if any, this permeation serves is still a valid question. Interestingly, in macrophage-derived RAW264 cells, the anionic dye uptake appears to be the principal P2X7R-induced uptake response, because it is much faster than the cationic dye uptake. (In the study by Cankurtaran-Sayar et al. (2009), compare the normalized YoPro-1 uptake shown in the supplements to the normalized lucifer yellow uptake.) Here, it can be hypothesized that this anionic pathway that can carry the anionic dyes both ways (our unpublished results in RAW264 cells) is a candidate for the P2X7R-activated release mechanism for the anionic neurotransmitters such as glutamate by astrocytes described in Duan et al. (2003) and Fellin et al. (2006) and may be considered as a separate potential drug target.

Additionally, as mentioned earlier, ATP is thought to be an important mediator of inflammation, and P2X7R-activated $\mathrm{ATP}^{4-}$ release acts as a positive feedback mechanism in inflammatory processes. This release was proposed to be through the P2X7R ionic channel itself (Pellegatti et al., 2005; also see Di Virgilio et al., 2017), which is clearly in direct conflict with the cation-selective nature of the P2X7R pore. The P2X7Ractivated separate anion-specific pathway hypothesized earlier may solve this problem. Whether the pannexin protein (as suggested by Di Virgilio et al., 2017) or another-as yet unidentified-protein forms this pathway still needs to be answered.

Even if the role of pannexin in cationic large molecule permeation has lost ground, its involvement in the P2X7R action as an anionic conduit is still a possibility that should be investigated. On the other hand, it is difficult to form a consistent hypothesis about P2X7R-induced ATP release for the time being, as it can also be seen in P2X7Rexpressing HEK293 cells (Pellegatti et al., 2005) where the anionic dye uptake is not observed (Schachter et al., 2008; Cankurtaran-Sayar et al., 2009). Definitely, more work is needed to clearly understand these results. Whether the P2X7R-induced large-cation-permeability has any function is also an interesting question that requires further investigation.

Mechanism 3. Does P2X7R Directly Interact with Cellular Signaling Pathways? While the mechanism of large molecule permeabilization had been the center of discussion, the literature contains intriguing but mostly neglected data possibly pointing at another mechanism of action for P2X7R, which seems to be distinct from the activation of permeation pathways. There are numerous studies showing that P2X7R stimulation leads to activation of a diverse range of intracellular signaling molecules such as protein kinases or phospholipases. However, in comparison, there are very few studies designed to test whether the activation of these signaling molecules are due to mechanisms related to the ion channel activity of the receptor such as intracellular calcium increase, depolarization or, as discussed earlier, depletion of intracellular $\mathrm{K}^{+}$.

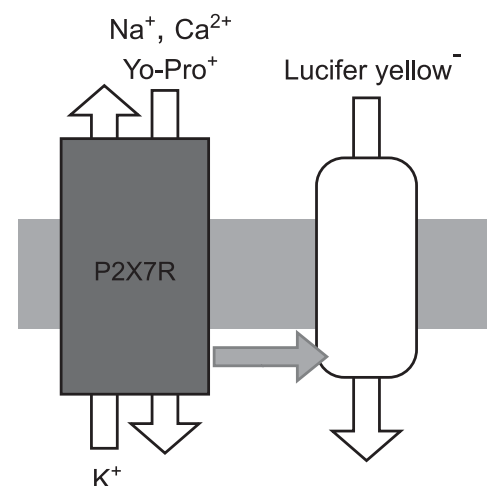

Model 1: P2X7R is a cation channel permeable to large cationic molecules. It is also coupled to an accessory pathway for anionic large molecules.

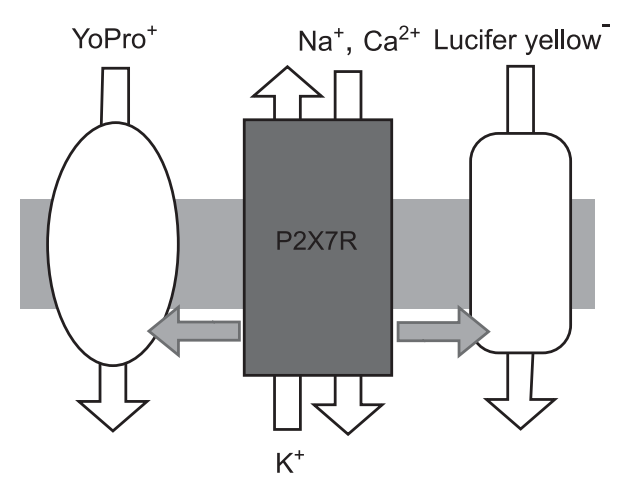

Model 2: P2X7R is a cation channel and is also coupled to (at least) two accessory pathays; one for cationic the other for anionic large molecules.
Fig. 2. Basic models to explain the available data about the P2X7R-induced cell membrane permeabilization. P2X7R protein is shown as a gray box. White boxes are the proposed accessory cationic or anionic permeation pathways activated by P2X7R. Horizontal gray arrows indicate activation by P2X7R. YoPro and lucifer yellow are shown as representative cationicand anionic-large molecules, respectively, as they are the commonly used experimental tracers. All the boxes represent passive transportation pathways where the actual flow directions will be dependent on the electrochemical potential of each ion. Vertical white arrows, in turn, indicate the expected flow directions of the indicated ions in commonly used experimental conditions. 
Yet the existing data provide enough evidence to consider the possibility that P2X7R may not only act as an ion channel but also, akin to metabotropic receptors, may directly interact with and activate intracellular signaling proteins. The well-known P2X7R-activated blebbing response, for example, which has been described both in macrophages and HEK293 expression systems (Virginio et al., 1999a; Morelli et al., 2003; Verhoef et al., 2003; Pfeiffer et al., 2004), seems to require activation of protein kinases (Morelli et al., 2003; Verhoef et al., 2003; Pfeiffer et al., 2004; Mackenzie et al., 2005) and is independent of extracellular $\mathrm{Ca}^{2+}$ (Verhoef et al., 2003; Pfeiffer et al., 2004), or at least has a $\mathrm{Ca}^{2+}$-independent mode (Mackenzie et al., 2005). The results of the study by Morelli et al. (2003) show that $\mathrm{Ca}^{2+}$ uptake is necessary but not sufficient for P2X7Rinduced blebbing (Morelli et al., 2003).

A receptor pharmacologically similar to $\mathrm{P} 2 \mathrm{X} 7 \mathrm{R}$ can activate phospholipase D enzyme (PLD) in BAC1.2F5 macrophages phospholipase A2 enzyme (PLA2) in submandibular glands (el-Moatassim and Dubyak, 1992; Alzola et al., 1998), again without the involvement of extracellular $\mathrm{Ca}^{2+}$ (There is also a $\mathrm{Ca}^{2+}$-dependent component of the P2X7R-induced phospholipase activation in some cells, which can be discerned by using different phospholipase inhibitors [Gargett et al., 1996; Alzola et al., 1998].) Wholecell and cell-attached patch experiments in smooth muscle cells convincingly have shown that stimulation of a native ATP receptor with a pharmacologic and electrophysiologic profile similar to P2X7R (Ugur et al., 1997) activates some $\mathrm{K}^{+}$channels through a soluble intracellular messenger independently from $\mathrm{K}^{+}$efflux or $\mathrm{Ca}^{2+}$ influx (Zou et al., 2001). Uptake of anionic dyes, the response discussed in detail earlier, seems to require activation of a separate pathway, which is clearly not mediated by intracellular $\mathrm{Ca}^{2+}$ increase, or $\mathrm{K}^{+}$loss in RAW264 cells (Cankurtaran-Sayar et al., 2009). Similarly, an inhibitory interaction between P2X2R and Ni-AchR $(\alpha 3 \beta 4)$, which was clearly independent of factors such as $\mathrm{Ca}^{2+}$ influx or depolarization, has also been reported in an oocyte expression system (Khakh et al., 2000).

Finally, P2X7R seems to interact with intracellular signal transduction proteins such as 1-phosphatidylinositol 4-kinase enzyme, receptor-type protein tyrosine phosphatase- $\beta$, or epithelial membrane proteins (EMPs 1, 2, and 3) within large molecular complexes, The term "P2X7R signaling complex" has been coined for this proposed structure where P2X7R activity also seems to be regulated by its protein partners as a feedback mechanism (Kim et al., 2001; Wilson et al., 2002). There are also reports indicating that P2X7R may coimmunoprecipitate with proteins of the NLRP inflammasome complex (Minkiewicz et al., 2013; Franceschini et al., 2015). If these findings indicate a direct interaction between P2X7R and the NLRP complex as suggested, they may add a further layer of complication to the processes such as P2X7R activated IL- $1 \beta$ secretion. Definitely, more studies are needed to confirm and expand these observations.

Nevertheless, the available data clearly suggest that neither the ion channel activity nor the permeability to large molecules adequately explain all the actions of P2X7R, and the receptor may directly interact with intracellular signaling proteins. (Fig. 1 working hypothesis 3 .) Recently, similar "metabotropic"-like actions have been proposed for other well-known ligand-gated ion channel receptors (for reviews, see Valbuena and Lerma, 2016; Kabbani and Nichols, 2018).

\section{Conclusions/Future Perspectives}

In this review, we discussed examples of P2X7R-related phenomena in the framework of three working hypotheses with the objective of reaching a better understanding in the P2X7R field as a whole. It should be noted that all three hypotheses discussed in this review are working hypotheses. Although they are based on experimental results, especially the last two still require further experimental testing to be firmly established.

As the examples we have discussed illustrate, approaching the P2X7R responses from a mechanistic point of view is eventually a key factor for a clear understanding of how P2X7R works. Experiments cautiously designed to discern which of the discussed mechanisms is actually at work in each individual case will certainly improve and expand our vision. In our view, the most interesting and promising clues will be obtained from the experiments aimed to dissect the cation channel-related action mechanisms of the P2X7R (i.e., depolarization, $\mathrm{K}^{+}$efflux, or $\mathrm{Ca}^{2+}$ influx) from its presumed direct interactions with intracellular signaling proteins. Clearly, careful experimental designs are required to do this dissection as both the intracellular $\mathrm{K}^{+}$depletion and the $\mathrm{Ca}^{2+}$ increase are well-established activators of cellular responses.

Another equally important point here is the elimination of the contamination from other purinergic receptors (other P2Xs, more importantly P2Ys and also adenosine receptors), especially when the experiments are performed in native cells that potentially express various types of purinergic receptors. As in the case of IL- $1 \beta$ processing, even a seemingly simple mechanism such as $\mathrm{K}^{+}$depletion, which is clearly P2X7R dependent, can harbor various difficulties. Additional essential components such as other ion channels and other ATP receptors may come into play, creating a complicated situation. Also it should always be kept in mind that other P2X receptors may activate P2X7R-like responses, as in the case of a combination of P2X2 and P2X5 receptors which can mimic some responses of the P2X7R (Compan et al., 2012).

The mechanisms of most of the P2X7R-induced responses such as promotion of cell growth, IL- $1 \beta$ processing and secretion, activation of anionic transport pathways, and membrane blebbing are not fully understood. In addition to the examples we have provided, P2X7R was proposed to activate phosphatidylinositol-4,5-bisphosphate 3-kinase (Amoroso et al., 2015), nuclear factor $\kappa \mathrm{B}$ (Ferrari et al., 1997b; Korcok et al., 2004), PLA2 and PLD (Panupinthu et al., 2008), AKT (Xia et al., 2015), and phosphorylates glycogen synthase kinase $3 \beta$ (Amoroso et al., $2015)$ in various cell types. For more examples the reader is referred to Di Virgilio and Adinolfi (2017).

Yet this plethora of data, except for a few cases, gives almost no insight into the mechanisms linking the P2X7R stimulation to the activation of these proteins. These signaling proteins are known to play important roles in cancer, inflammation, and many other cellular responses. The question as to whether activation of $\mathrm{K}^{+}, \mathrm{Na}^{+}$, or the $\mathrm{Ca}^{2+}$ fluxes is sufficient or a direct activation of intracellular signaling protein(s) is also 
involved in all these P2X7R-induced responses remains at the core of the P2X7R field. If the latter case is true then the next question will be the identity of these signaling proteins. In this regard, the proteins proposed to be P2X7R signaling partners in sP2X7R signaling complexes (Kim et al., 2001; Wilson et al., 2002; Minkiewicz et al., 2013; Franceschini et al., 2015) can be good candidates to start with. Resolving this point will not only drastically improve our understanding about the role of this receptor in cancer, inflammation, and many other fields but may also significantly change our perception about the receptor actions in general.

\section{Acknowledgments}

We wish to thank Dr. Ongun Onaran for insightful discussion and also critically reading the paper, Şule Girmen for correcting the manuscript.

\section{Authorship Contributions}

Wrote or contributed to the writing of the manuscript: M. Ugur, Ö. Ugur.

\section{References}

Adinolfi E, Callegari MG, Ferrari D, Bolognesi C, Minelli M, Wieckowski MR, Pinton P, Rizzuto R, and Di Virgilio F (2005) Basal activation of the P2X7 ATP receptor elevates mitochondrial calcium and potential, increases cellular ATP levels, and promotes serum-independent growth. Mol Biol Cell 16:3260-3272.

Adinolfi E, Cirillo M, Woltersdorf R, Falzoni S, Chiozzi P, Pellegatti P, Callegari MG, Sandonà D, Markwardt F, Schmalzing G, et al. (2010) Trophic activity of a naturally occurring truncated isoform of the $\mathrm{P} 2 \mathrm{X} 7$ receptor. FASEB $J$ 24:3393-3404.

Adinolfi E, Giuliani AL, De Marchi E, Pegoraro A, Orioli E, and Di Virgilio F (2018) The P2X7 receptor: a main player in inflammation. Biochem Pharmaco 151:234-244

Adinolfi E, Raffaghello L, Giuliani AL, Cavazzini L, Capece M, Chiozzi P, Bianchi G, Kroemer G, Pistoia V, and Di Virgilio F (2012) Expression of P2X7 receptor increases in vivo tumor growth Cancer Res 72:2957-2969.

Alberto AV, Faria RX, Couto CG, Ferreira LG, Souza CA, Teixeira PC, Fróes MM, and Alves LA (2013) Is pannexin the pore associated with the P2X7 receptor? Naunyn Schmiedebergs Arch Pharmacol 386:775-787.

Alzola E, Pérez-Etxebarria A, Kabré E, Fogarty DJ, Métioui M, Chaïb N, Macarulla JM, Matute C, Dehaye JP, and Marino A (1998) Activation by P2X7 agonists of two phospholipases A2 (PLA2) in ductal cells of rat submandibular gland. Coupling of the calcium-independent PLA2 with kallikrein secretion. J Biol Chem 273 30208-30217.

Amoroso F, Capece M, Rotondo A, Cangelosi D, Ferracin M, Franceschini A, Raffaghello L, Pistoia V, Varesio L, and Adinolfi E (2015) The P2X7 receptor is a key modulator of the PI3K/GSK3 $\beta /$ VEGF signaling network: evidence in experimental neuroblastoma. Oncogene 34:5240-5251.

Banke TG, Chaplan SR, and Wickenden AD (2010) Dynamic changes in the TRPA1 selectivity filter lead to progressive but reversible pore dilation. Am J Physiol Cell Physiol 298:C1457-C1468.

Boldt W, Klapperstück M, Büttner C, Sadtler S, Schmalzing G, and Markwardt F (2003) Glu496Ala polymorphism of human P2X7 receptor does not affect its electrophysiological phenotype. Am J Physiol Cell Physiol 284:C749-C756.

Browne LE, Compan V, Bragg L, and North RA (2013) P2X7 receptor channels allow direct permeation of nanometer-sized dyes. J Neurosci 33:3557-3566.

Cankurtaran-Sayar S, Sayar K, and Ugur M (2009) P2X7 receptor activates multiple selective dye-permeation pathways in RAW 264.7 and human embryonic kidney 293 cells. Mol Pharmacol 76:1323-1332.

Chung MK, Güler AD, and Caterina MJ (2008) TRPV1 shows dynamic ionic selectivity during agonist stimulation. Nat Neurosci 11:555-564.

Cockcroft S and Gomperts BD (1980) The ATP4- receptor of rat mast cells. Biochem $J$ 188:789-798.

Compan V, Ulmann L, Stelmashenko O, Chemin J, Chaumont S, and Rassendren F (2012) P2X2 and P2X5 subunits define a new heteromeric receptor with P2X7-like properties. J Neurosci 32:4284-4296.

Di A Xiong S, Ye Z, Malireddi RKS, Kometani S, Zhong M, Mittal M, Hong Z Kanneganti TD, Rehman J, et al. (2018) The TWIK2 potassium efflux channel in macrophages mediates NLRP3 inflammasome-induced inflammation. Immunity 49:56-65.e4

Di Virgilio F and Adinolfi E (2017) Extracellular purines, purinergic receptors and tumor growth. Oncogene 36:293-303.

Di Virgilio F, Dal Ben D, Sarti AC, Giuliani AL, and Falzoni S (2017) The P2X7 receptor in infection and inflammation. Immunity 47:15-31.

Di Virgilio F, Schmalzing G, and Markwardt F (2018) The elusive P2X7 macropore. Trends Cell Biol 28:392-404.

Duan S, Anderson CM, Keung EC, Chen Y, Chen Y, and Swanson RA (2003) P2X7 receptor-mediated release of excitatory amino acids from astrocytes. J Neurosci $\mathbf{2 3}$ $1320-1328$

El-Moatassim C and Dubyak GR (1992) A novel pathway for the activation of phospholipase $\mathrm{D}$ by $\mathrm{P} 2 \mathrm{z}$ purinergic receptors in BAC1.2F5 macrophages. $J$ Biol Chem 267:23664-23673.
Fellin T, Pozzan T, and Carmignoto G (2006) Purinergic receptors mediate two distinct glutamate release pathways in hippocampal astrocytes. J Biol Chem 281: $4274-4284$

Ferrari D, Chiozzi P, Falzoni S, Dal Susino M, Melchiorri L, Baricordi OR, and Di Virgilio F (1997a) Extracellular ATP triggers IL-1 beta release by activating the purinergic P2Z receptor of human macrophages. $J$ Immunol 159:1451-1458.

Ferrari D, Pizzirani C, Adinolfi E, Lemoli RM, Curti A, Idzko M, Panther E, and Di Virgilio F (2006) The P2X7 receptor: a key player in IL-1 processing and release. J Immunol 176:3877-3883.

Ferrari D, Wesselborg S, Bauer MKA, and Schulze-Osthoff K (1997b) Extracellular ATP activates transcription factor NF- $\kappa$ B through the P2Z purinoreceptor by selectively targeting NF- $\kappa$ B p65. J Cell Biol 139:1635-1643.

Franceschini A, Capece M, Chiozzi P, Falzoni S, Sanz JM, Sarti AC, Bonora M Pinton P, and Di Virgilio F (2015) The P2X7 receptor directly interacts with the NLRP3 inflammasome scaffold protein. FASEB $J$ 29:2450-2461.

Gargett CE, Cornish EJ, and Wiley JS (1996) Phospholipase D activation by P2Zpurinoceptor agonists in human lymphocytes is dependent on bivalent cation influx. Biochem J 313:529-535.

Greenberg S, Di Virgilio F, Steinberg TH, and Silverstein SC (1988) Extracellular nucleotides mediate $\mathrm{Ca}^{2+}$ fluxes in J774 macrophages by two distinct mechanisms. $J$ Biol Chem 263:10337-10343.

Gu BJ, Zhang W, Worthington RA, Sluyter R, Dao-Ung P, Petrou S, Barden JA, and Wiley JS (2001) A Glu-496 to Ala polymorphism leads to loss of function of the human P2X7 receptor. J Biol Chem 276:11135-11142.

Hanley PJ, Kronlage M, Kirschning C, del Rey A, Di Virgilio F, Leipziger J, Chessell IP, Sargin S, Filippov MA, Lindemann O, et al. (2012) Transient P2X7 receptor activation triggers macrophage death independent of Toll-like receptors 2 and 4 caspase-1, and pannexin-1 proteins. J Biol Chem 287:10650-10663.

Harkat M, Peverini L, Cerdan AH, Dunning K, Beudez J, Martz A, Calimet N, Specht A, Cecchini M, Chataigneau T, et al. (2017) On the permeation of large organic cations through the pore of ATP-gated P2X receptors. Proc Natl Acad Sci USA 114:E3786-E3795.

Jacques-Silva MC, Rodnight R, Lenz G, Liao Z, Kong Q, Tran M, Kang Y, Gonzalez FA, Weisman GA, and Neary JT (2004) P2X7 receptors stimulate AKT phosphorylation in astrocytes. $\mathrm{Br}$ J Pharmacol 141:1106-1117.

Jiang LH, Rassendren F, Mackenzie A, Zhang YH, Surprenant A, and North RA (2005) N-methyl-D-glucamine and propidium dyes utilize different permeation pathways at rat P2X(7) receptors. Am J Physiol Cell Physiol 289:C1295-C1302.

Kabbani N and Nichols RA (2018) Beyond the channel: metabotropic signaling by nicotinic receptors. Trends Pharmacol Sci 39:354-366.

Karasawa A, Michalski K, Mikhelzon P, and Kawate T (2017) The P2X7 receptor forms a dye-permeable pore independent of its intracellular domain but dependent on membrane lipid composition. eLife 6; 31186

Khakh BS, Bao XR, Labarca C, and Lester HA (1999) Neuronal P2X transmitter-gated cation channels change their ion selectivity in seconds. Nat Neurosci 2:322-330.

Khakh BS, Zhou X, Sydes J, Galligan JJ, and Lester HA (2000) State-dependent cross-inhibition between transmitter-gated cation channels. Nature 406:405-410.

Kim M, Jiang LH, Wilson HL, North RA, and Surprenant A (2001) Proteomic and functional evidence for a P2X7 receptor signalling complex. EMBO J 20:6347-6358.

Korcok J, Raimundo LN, Ke HZ, Sims SM, and Dixon SJ (2004) Extracellular nucleotides act through P2X7 receptors to activate NF- $\kappa \mathrm{B}$ in osteoclasts. $J$ Bone Miner Res 19:642-651.

Kubick C, Schmalzing G, and Markwardt F (2011) The effect of anions on the human P2X7 receptor. Biochim Biophys Acta 1808:2913-2922.

Le Stunff H and Raymond MN (2007) P2X7 receptor-mediated phosphatidic acid production delays ATP-induced pore opening and cytolysis of RAW 264.7 macrophages. Cell Signal 19:1909-1918.

Li M, Toombes GE, Silberberg SD, and Swartz KJ (2015) Physical basis of apparent pore dilation of ATP-activated P2X receptor channels. Nat Neurosci 18:1577-1583.

Mackenzie AB, Young MT, Adinolfi E, and Surprenant A (2005) Pseudoapoptosis induced by brief activation of ATP-gated P2X7 receptors. J Biol Chem 280: 33968-33976.

Minkiewicz J, de Rivero Vaccari JP, and Keane RW (2013) Human astrocytes express a novel NLRP2 inflammasome. Glia 61:1113-1121.

Morelli A, Chiozzi P, Chiesa A, Ferrari D, Sanz JM, Falzoni S, Pinton P, Rizzuto R, Olson MF, and Di Virgilio F (2003) Extracellular ATP causes ROCK I-dependent bleb formation in P2X7-transfected HEK293 cells. Mol Biol Cell 14:2655-2664.

Panupinthu N, Rogers JT, Zhao L, Solano-Flores LP, Possmayer F, Sims SM, and Dixon SJ (2008) P2X7 receptors on osteoblasts couple to production of lysophosphatidic acid: a signaling axis promoting osteogenesis. J Cell Biol 181:859-871.

Pellegatti P, Falzoni S, Pinton P, Rizzuto R, and Di Virgilio F (2005) A novel recombinant plasma membrane-targeted luciferase reveals a new pathway for ATP secretion. Mol Biol Cell 16:3659-3665.

Pelegrin P and Surprenant A (2006) Pannexin-1 mediates large pore formation and interleukin-1 $\beta$ release by the ATP-gated P2X7 receptor. EMBO J 25:5071-5082.

Petrou S, Ugur M, Drummond RM, Singer JJ, and Walsh JV Jr (1997) P2X7 purinoceptor expression in Xenopus oocytes is not sufficient to produce a pore-forming P2Z-like phenotype. FEBS Lett 411:339-345.

Peverini L, Beudez J, Dunning K, Chataigneau T, and Grutter T (2018) New insights into permeation of large cations through ATP-gated P2X receptors. Front Mol Neurosci 11:265.

Pfeiffer ZA, Aga M, Prabhu U, Watters JJ, Hall DJ, and Bertics PJ (2004) The nucleotide receptor P2X7 mediates actin reorganization and membrane blebbing in RAW 264.7 macrophages via p38 MAP kinase and Rho. J Leukoc Biol 75:1173-1182.

Pippel A, Stolz M, Woltersdorf R, Kless A, Schmalzing G, and Markwardt F (2017) Localization of the gate and selectivity filter of the full-length P2X7 receptor. Proc Natl Acad Sci USA 114:E2156-E2165.

Qu Y, Misaghi S, Newton K, Gilmour LL, Louie S, Cupp JE, Dubyak GR, Hackos D, and Dixit VM (2011) Pannexin-1 is required for ATP release during apoptosis but not for inflammasome activation. J Immunol 186:6553-6561. 
Riedel T, Lozinsky I, Schmalzing G, and Markwardt F (2007a) Kinetics of P2X7 receptor-operated single channels currents. Biophys $J$ 92:2377-2391.

Riedel T, Schmalzing G, and Markwardt F (2007b) Influence of extracellular monovalent cations on pore and gating properties of P2X7 receptor-operated singlechannel currents. Biophys $J$ 93:846-858.

Rozengurt E, Heppel LA, and Friedberg I (1977) Effect of exogenous ATP on the permeability properties of transformed cultures of mouse cell lines. J Biol Chem 252:4584-4590.

Schachter J, Motta AP, de Souza Zamorano A, da Silva-Souza HA, Guimarães MZ, and Persechini PM (2008) ATP-induced P2X7-associated uptake of large molecules involves distinct mechanisms for cations and anions in macrophages. J Cell Sci 121:3261-3270.

Smart ML, Gu B, Panchal RG, Wiley J, Cromer B, Williams DA, and Petrou S (2003) $\mathrm{P} 2 \mathrm{X} 7$ receptor cell surface expression and cytolytic pore formation are regulated by a distal C-terminal region. J Biol Chem 278:8853-8860.

Solle M, Labasi J, Perregaux DG, Stam E, Petrushova N, Koller BH, Griffiths RJ, and Gabel CA (2001) Altered cytokine production in mice lacking P2X(7) receptors. $J$ Biol Chem 276:125-132.

Steinberg TH, Newman AS, Swanson JA, and Silverstein SC (1987) ATP4- permeabilizes the plasma membrane of mouse macrophages to fluorescent dyes. J Biol Chem 262:8884-8888.

Surprenant A, Rassendren F, Kawashima E, North RA, and Buell G (1996) The cytolytic P2Z receptor for extracellular ATP identified as a P2X receptor (P2X7). Science 272:735-738.

Tatham PE and Lindau M (1990) ATP-induced pore formation in the plasma membrane of rat peritoneal mast cells. J Gen Physiol 95:459-476.

Ugur M, Drummond RM, Zou H, Sheng P, Singer JJ, and Walsh JV Jr (1997) An ATP-gated cation channel with some P2Z-like characteristics in gastric smooth muscle cells of toad. J Physiol 498:427-442.
Valbuena S and Lerma J (2016) Non-canonical signaling, the hidden life of ligandgated ion channels. Neuron 92:316-329.

Verhoef PA, Estacion M, Schilling W, and Dubyak GR (2003) P2X7 receptordependent blebbing and the activation of Rho-effector kinases, caspases, and IL-1 beta release. J Immunol 170:5728-5738.

Virginio C, MacKenzie A, North RA, and Surprenant A (1999a) Kinetics of cell lysis, dye uptake and permeability changes in cells expressing the rat P2X7 receptor J Physiol 519:335-346.

Virginio C, MacKenzie A, Rassendren FA, North RA, and Surprenant A (1999b) Pore dilation of neuronal P2X receptor channels. Nat Neurosci 2:315-321.

Walev I, Reske K, Palmer M, Valeva A, and Bhakdi S (1995) Potassium-inhibited processing of IL-1 beta in human monocytes. EMBO $J$ 14:1607-1614

Wilson HL, Wilson SA, Surprenant A, and North RA (2002) Epithelial membrane proteins induce membrane blebbing and interact with the $\mathrm{P} 2 \mathrm{X} 7$ receptor $\mathrm{C}$ terminus. $J$ Biol Chem 277:34017-34023.

Xia J, Yu X, Tang L, Li G, and He T (2015) P2X7 receptor stimulates breast cancer cell invasion and migration via the AKT pathway. Oncol Rep 34:103-110.

Yan Z, Li S, Liang Z, Tomić M, and Stojilkovic SS (2008) The P2X7 receptor channel pore dilates under physiological ion conditions. J Gen Physiol 132:563-573.

Young CNJ and Górecki DC (2018) P2RX7 Purinoceptor as a therapeutic target-the second coming? Front Chem 6:248.

Zou H, Ugur M, Drummond RM, and Singer JJ (2001) Coupling of a P2Z-like purinoceptor to a fatty acid-activated $\mathrm{K}(+)$ channel in toad gastric smooth muscle cells. J Physiol 534:59-70.

Address correspondence to: Dr. Mehmet Ugur, Faculty of Medicine, Department of Biophysics, Ankara University, A. Adnan Saygun Cd., 06230 Ankara, Turkey. E-mail: mugur@medicine.ankara.edu.tr 